\title{
Topographic Spread of Inferior Colliculus Activation in Response to Acoustic and Intracochlear Electric Stimulation
}

\author{
Russell L. Snyder, ${ }^{1}$ Julie A. Bierer, ${ }^{1}$ And John C. Middlebrooks ${ }^{2}$ \\ ${ }^{1}$ Epstein Laboratory, Department of Otolaryngology, University of California, San Francisco, CA 94143-0526, USA \\ ${ }^{2}$ Kresge Hearing Research Institute, Neuroscience Program, University of Michigan, Ann Arbor, MI 48109-0506, USA
}

Received: 17 July 2003; Accepted: 5 April 2004; Online publication: 12 August 2004

\section{ABSTRACT}

The design of contemporary multichannel cochlear implants is predicated on the presumption that they activate multiple independent sectors of the auditory nerve array. The independence of these channels, however, is limited by the spread of activation from each intracochlear electrode across the auditory nerve array. In this study, we evaluated factors that influence intracochlear spread of activation using two types of intracochlear electrodes: (1) a clinical-type device consisting of a linear series of ring contacts positioned along a silicon elastomer carrier, and (2) a pair of visually placed (VP) ball electrodes that could be positioned independently relative to particular intracochlear structures, e.g., the spiral ganglion. Activation spread was estimated by recording multineuronal evoked activity along the cochleotopic axis of the central nucleus of the inferior colliculus (ICC). This activity was recorded using silicon-based singleshank, 16-site recording probes, which were fixed within the ICC at a depth defined by responses to acoustic tones. After deafening, electric stimuli consisting of single biphasic electric pulses were presented with each electrode type in various stimulation configurations (monopolar, bipolar, tripolar) and/or various electrode orientations (radial, off-radial, longitudinal). The results indicate that monopolar (MP) stimulation with either electrode type produced widepread excitation across the ICC. Bipolar (BP)

Correspondence to: Russell L. Snyder • Epstein Laboratory • Box 0526, U490 - University of California - San Francisco, CA 94143-0526. Telephone: (415) 476-1726; fax: (415) 476-2169; email: rsnyder@ itsa.ucsf.edu stimulation with banded pairs of electrodes oriented longitudinally produced activation that was somewhat less broad than MP stimulation, and tripolar (TP) stimulation produced activation that was more restricted than MP or BP stimulation. Bipolar stimulation with radially oriented pairs of VP ball electrodes produced the most restricted activation. The activity patterns evoked by radial VP balls were comparable to those produced by pure tones in normal-hearing animals. Variations in distance between radially oriented VP balls had little effect on activation spread, although increases in interelectrode spacing tended to reduce thresholds. Bipolar stimulation with longitudinally oriented VP electrodes produced broad activation that tended to broaden as the separation between electrodes increased.

Keywords: cochlear implant, cochlear prosthesis, deafness, auditory nervous system, multichannel recording, auditory prosthesis

\section{INTRODUCTION}

Multichannel cochlear implants (CIs) have become the standard of care for severely to profoundly deaf adults and children. They consist of arrays of intracochlear electrodes connected to a multichannel extracochlear sound processor. The output of each processor channel is distributed to one electrode channel, i.e., one contact or bipolar pair of contacts, located at a tonotopically appropriate cochlear place. Ideally, the electric signal at each cochlear site would 
activate a restricted, tonotopically appropriate, and largely independent population of auditory nerve fibers. Clinical and experimental psychophysical results, however, suggest that CI electrode channels often activate broadly overlapping and in some cases inappropriate neural populations (Eddington et al. 1978; Shannon 1983; Townshend et al. 1987; Collins et al. 1997). Broad activation of neural populations leads to functional interaction among implant channels. Activation of inappropriate neural populations produces assignment of acoustic information to inappropriate cochlear locations. Therefore, one means of improving cochlear implant performance might be to control more accurately the spread and location of auditory neural activation.

Studies of the spread of auditory nerve activation in response to electric stimuli have involved clinical and psychophysical studies in humans (Tong and Clark 1986; Lim et al. 1989; Chatterjee and Shannon 1998; Hanekom and Shannon 1997), electrophysiological studies in animals (Woolsey and Walzl 1943; Van den Honert and Stypulkowski 1987; Snyder et al. 1990, 1991; Leake et al. 1991, 1995, 2000; Kral et al. 1998; Shepherd et al. 1999; Rebscher et al. 2001; Taniguchi et al. 1997; Raggio and Schreiner. 1999; Bierer and Middlebrooks 2002; Middlebrooks and Bierer 2002), and theoretical modeling studies (Finley 1989; Finley et al. 1987, 1990; Frijns et al. 1994, 1995, 1996; Rattay et al. 2001a,b). Previous studies have been limited by a number of technical problems. In most human studies, for example, the precise locations of stimulating electrodes along the cochlear spiral and their positions relative to excitable neural elements are not known. Moreover, responses evoked by acoustic stimuli cannot be compared with those evoked by various electrode configurations in single subjects, most of whom are severely or profoundly deaf bilaterally prior to implantation. In addition, tests of various electrode configurations are constrained by the design of the implanted arrays and their processors. In most animal studies, the numbers of electric stimulus configurations that can be tested are limited by the difficulty of repeating stimulus sets (acoustic and electric) for multiple recording sites and by the difficulties involved in sequential recordings of neurons at multiple sites.

In the present report, we describe results from an animal model in which we compared the responses of a constant central neural population to a variety of acoustic and electric stimuli. Neural responses were recorded simultaneously at a fixed array of 16 locations in the inferior colliculus (IC) of anesthetized guinea pigs. We chose to record from the IC because its tonotopic representation allows simultaneous sampling of activity across a large part of the fre- quency representation. In each animal, we first recorded the responses to acoustic tones and noise bursts in a normal-hearing condition, thereby identifying response properties of the selected ICC neurons distributed along its tonotopic organization. Then we fixed the recording probe in place, exposed the contralateral cochlea, and deafened it with an injection of an ototoxic drug. Finally, we recorded responses to electric stimuli presented through a clinical-style cochlear implant or through visually placed (VP) ball electrodes positioned on specific intracochlear structures. Since the recording electrodes were fixed in place throughout all acoustic and electric stimulation, responses could be interpreted with reference to the tonotopic representation identified under normal-hearing conditions.

These results using both a longitudinal array of banded electrodes and VP ball electrodes permit the following conclusions: (1) Spread of activation by a radial bipolar electrode pair is nearly as restricted as the spread of activation elicited by a pure tone. (2) Spread of activation along the tonotopic axis increases in order from radial bipolar, to longitudinal tripolar, to longitudinal bipolar, to monopolar. (3) Spatial separation and precise location of radial bipolar electrode pairs influence thresholds but has little influence on the spread of activation. (4) Banded clinical-style electrodes, when activated in a longitudinal tripolar configuration, were nearly as selective as visually placed bipolar electrodes that were activated in a radial configuration.

\section{METHODS}

\section{Anesthesia and surgery}

Data were collected from 10 healthy adult pigmented guinea pigs (400-700 g). Nine of these animals had normal hearing at the beginning of the experiments. An additional animal was deafened with an intracochlear injection of neomycin sulfate one week prior to the experiment. The results from this animal were qualitatively indistinguishable from those obtained from the acutely deafened animals. Animals were initially sedated with a subcutaneous injection of ketamine hydrochloride $(40 \mathrm{mg} / \mathrm{kg})$ and xylazine (10 $\mathrm{mg} / \mathrm{kg})$. Additional intramuscular injections of a 9:1 mixture of ketamine/xylazine were given as needed to maintain an areflexive state. Heart rate, respiratory rate, and body temperature were monitored continuously. Core body temperature was maintained at $38^{\circ} \mathrm{C}$ with a thermostatically controlled heating pad. A tracheal cannula was inserted to insure an unobstructed airway. A dorsal midline incision was made in the scalp to expose the dorsal surface of the skull. The head was fixed in place by a phenolic rod that 
was attached to the skull anterior to the bregma using small self-tapping screws and dental acrylic. The right temporalis muscle was reflected, and a 5-mm opening was made in the right parietal bone just dorsal to the parietal/temporal suture and just rostral to the tentorium. The dura was incised and reflected to expose the lateral and posterior occipital cortex on the right side. This cortex was aspirated to allow direct visualization of the dorsal and lateral surface of the IC.

Once the right IC was visualized, a Teflon-coated silver wire was inserted and fixed behind the IC to serve as a reference electrode for both auditory brainstem response (ABR) and ICC neuronal recordings. Then a silicon-substrate, thin-film, multichannel recording probe (Center for Neural Communication Technology, Ann Arbor, MI; Drake et al. 1988; Najafi et al. 1985) was inserted into the center of the IC using a micromanipulator. The probe was mounted on a custom-built head-stage that was held by the micromanipulator. The probe was inserted into the IC on a dorsolateral to ventromedial trajectory at a $45^{\circ}$ angle off the parasagital plane in the coronal plane. Using this trajectory, the probe traversed the central nucleus of the IC (ICC) roughly orthogonal to its isofrequency laminae (Snyder et al. $1990,1991,2000)$. The recording probe had 16 recording sites $\left(177 \mathrm{~m}^{2} /\right.$ site $)$ along a single shank distributed at $100-\mu \mathrm{m}$ intervals (center to center). The shank was $15 \mu \mathrm{m}$ thick and $100 \mu \mathrm{m}$ wide at the most proximal site, tapering to a width of $15 \mu \mathrm{m}$ at the most distal. The impedances at each site were 1.5$4 \mathrm{M} \Psi$. In the guinea pig, the $1.5-\mathrm{mm}$ distance from the most distal to the most proximal recording site allowed simultaneous recording of responses from neurons sensitive to frequencies spanning approximately 4.5 octaves (1-24 kHz, see Fig. 3). Probes were advanced until the most distal site recorded activity from neurons with a best frequency (BF) of approximately $24 \mathrm{kHz}$. Once this location was reached, the cortical deficit was filled with warm $2 \%$ agar dissolved in Ringer's solution. When the agar had solidified, the surrounding parietal and temporal bones and the proximal shank of the recording probe were covered with a thick layer of dental acrylic sealing the bony deficit and fixing the probe in place. After probe fixation, acoustic responses from all probe sites were recorded again from these normal-hearing animals. Acoustic responses were evoked by contralateral tones (see below) at a minimum of three levels separated by a maximum of $20 \mathrm{~dB}$ (usually 20, 40, and $60 \mathrm{~dB}$ SPL). Tone frequencies were separated by $1 / 8$ octave and usually ranged from 2 to $32 \mathrm{kHz}$.

Once the probe had been fixed in place and the preliminary acoustic responses had been recorded, the animal was repositioned to allow access to the left cochlea contralateral to the IC recording site. After repositioning, a second series of acoustic responses was recorded. In every case, the position of the recording probe remained in place relative to the tonotopic map, demonstrating the stability of the preparation. The tonotopic mapping with sounds permitted a BF to be assigned to each probe recording site, effectively calibrating the sites and allowing an intracochlear location to be assigned to each site. After this second series of acoustic recordings, the left cochlear bulla was opened so that the round window could be visualized. A silver wire was placed in skin below the left ear. This wire served as the active electrode to record the click-evoked ABR. An electrode in the skin below the right ear served as a ground electrode and the silver wire placed behind the contralateral IC served as the reference electrode. The ABR threshold was determined and then the animals were deafened by injecting a $10 \%$ solution of neomycin sulfate into the cochlea through a small slit in the round window membrane. Infusion of neomycin was continued while recording click-evoked responses until the ABR threshold exceeded the output of our audio system, approximately $100 \mathrm{~dB}$ SPL.

After the animals had been deafened, a cochleostomy was made in the lateral wall of the left cochlea at the junction of the "hook" and first cochlear turn. In seven animals, a modified Nucleus banded electrode (Cochlear Corporation), which consisted of the six most distal bands of a human cochlear implant, was inserted into the scala tympani of the cochlea through the cochleostomy. The bands were spaced in 0.75-mm intervals, center-to-center, along the longitudinal dimension of the scala. After insertion of this banded electrode, electric stimuli were presented in various configurations and ICC responses were recorded. Electrode configurations included monopolar stimulation (an intracochlear "active" electrode activated against an extracochlear "return" electrode in the skin behind the stimulated ear), bipolar stimulation (an intracochlear active electrode activated against an adjacent intracochlear return electrode), and tripolar stimulation (an intracochlear active electrode activated referenced to two adjacent intracochlear electrodes serving as the return). After the response to activation of different intracochlear electrode contacts in different configurations (monopolar, bipolar, or tripolar) had been recorded, the banded electrode was removed and the cochleostomy was enlarged so that two insulated platinum/ iridium wires ending in 250- $\mu \mathrm{m}$ balls could be placed visually within the basal scala tympani using a dual micromanipulator. These VP ball contacts were placed at several locations within the basal and second cochlear turns. These electrodes were activated in several configurations including monopolar, radial 
bipolar, and longitudinal bipolar (see Figs. 8, 10, and 12). The spatial distribution of responses evoked by activation of each configuration across the 16 sites of the fixed probe as a function of stimulus intensity were recorded and represented as a spatial tuning curve (STC). All procedures were conducted in accordance with the policies of the University of Michigan's University Committee on Use and Care of Animals.

\section{Stimulus generation and calibration}

Acoustic signals. Experiments were controlled by an Intel-based personal computer. Acoustic stimuli were synthesized digitally using equipment from TuckerDavis Technologies (TDT; Gainesville, FL). The sample rate for audio output was $100 \mathrm{kHz}$ with 16-bit resolution. Experiments were conducted in a soundattenuating chamber. Sound stimuli were presented monaurally to the ear contralateral to the studied IC. A headphone enclosed in a small case was connected to a sound-delivery tube inserted into the external auditory meatus near the tympanic membrane and sealed in place using dental acrylic. The headphone was calibrated using 0.25 -in. condenser microphone (ACU Pacific, Inc.) and a 0.3-cc coupler. The coupler positioned the microphone diaphragm at a location that approximated the location of the guinea pig tympanic membrane. The resulting calibration table was used for online correction of the headphone response. Tone and broadband Gaussian noise bursts were $40 \mathrm{~ms}$ in duration. Tones were ramped on and off with 5-ms rise/fall times, and noise bursts had 0.5$\mathrm{ms}$ rise/fall times. Noise bursts had a passband of 1$30 \mathrm{kHz}$ with abrupt cutoffs. Sound levels of tones and noise bursts were equated for root-mean-squared power. Stimulus frequencies ranged from 1 to $32 \mathrm{kHz}$ in 1/8- to 1/3-octave steps. Levels ranged from 0 to as high as $90 \mathrm{~dB}$ SPL in 5-20-dB steps.

Electric signals. Electric stimuli were generated by an 8-channel digital-to-analog converter (TDT DA8) that controlled a custom-made optically isolated current source. Electric stimuli consisted of single biphasic, charge-balanced pulses. Pulse phase duration was varied among 160, 320, 640, and $2560 \mu \mathrm{s} /$ phase. Polarity was initially cathodal at the active electrode (the intracochlear electrode in monopolar configurations, the more basal electrode in bipolar configurations, and the central electrode in tripolar configurations). Stimuli were presented at a rate of $2 / \mathrm{s}$.

\section{Multichannel recording and spike sorting}

Multichannel probes permitted simultaneous recording of spike activity from 16 sites arrayed across the ICC. Signals from these sites were amplified with

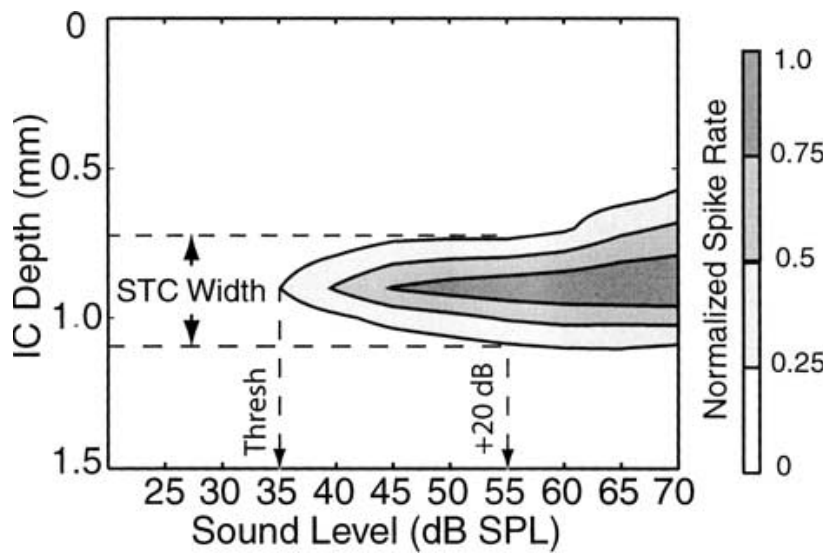

FIG. 1. Acoustic STC width calculation.

custom 16-channel pre- and postamplifiers, digitized at a $25-\mathrm{kHz}$ rate, sharply low-pass filtered below $6 \mathrm{kHz}$, resampled at a $12.5-\mathrm{kHz}$ sample rate, and then stored on the computer hard disk. Unit discharges were isolated using custom offline spike-sorting software (Furukawa et al. 2000) in three steps. First, digitized waveforms were analyzed to identify candidate spikes that exceeded criterion amplitude. Second, principal components analysis was used to represent those spikes in a two-dimensional visual display, i.e., each peak was represented by weights on the first two principal components. Clusters of spikes were defined by inspection of such plots and then used to generate template waveforms and acceptance limits. Third, the template and acceptance limits were used to screen all candidate spikes. Poststimulus times of accepted spikes were stored with $20-\mu$ s resolution. Well-isolated single units were recorded in many cases. In other cases, single units could not be isolated so we recorded multiunit clusters consisting of a small number of unresolved units, whose discharges were substantially larger than the background noise. In all cases, the stimulus selectivity of single-neuron and multineuronal responses was indistinguishable at the levels of analysis used in this study. For that reason, single-neuron and multineuronal responses were analyzed and presented identically. Recordings at particular sites were excluded from further analysis if units did not respond to an optimal stimulus with an average of at least 1 spike/trial or if the mean spike count changed by more than a factor of 2 over the recording period.

\section{Data analysis}

The results include data from individual recording sites as well as the distribution of activity across multiple recording sites. For individual recording sites, the lowest level that elicited a stimulus-locked response was defined as threshold. BF was defined as 
TABLE 1

Acoustic STC widths ${ }^{\mathrm{a}}$

\begin{tabular}{lccc}
\hline Acoustic stimulus & Mean width at $20 \mathrm{~dB}$ re threshold $(\mathrm{SD})$ & Number of animals & Number of valid STCs \\
\hline Pure tone & $381.9 \mu \mathrm{m}(144.2)$ & 8 & $52 / 60$ \\
BBN & $>1500 \mu \mathrm{m}$ & 5 & $0 / 5$ \\
\hline
\end{tabular}

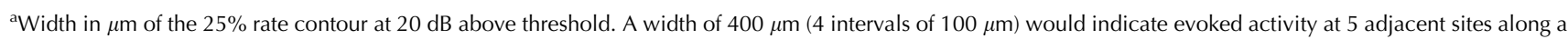
16 -site recording probe.

TABLE 2

Electric STC widths ${ }^{\text {a }}$

\begin{tabular}{lccc}
\hline Electrode configuration & Mean at $6 d B$ in $\mu \mathrm{m}(\mathrm{SD})$ & Number of animals & Number of valid STCs \\
\hline VP balls radial & $429.0(175.5)$ & 9 & $49 / 122$ \\
VP balls off-radial & $750.9(335.2)$ & 3 & $17 / 24$ \\
VP balls longitudinal & $718.5(207.2)$ & 5 & $6 / 18$ \\
VP balls monopolar & $>1500$ & 3 & $0 / 25$ \\
Banded tripolar & $547.7(58.4)$ & 3 & $4 / 16$ \\
Banded bipolar & $684.1(270.9)$ & 7 & $13 / 32$ \\
Banded monopolar & $>1500$ & $6 / 84$ \\
\hline
\end{tabular}

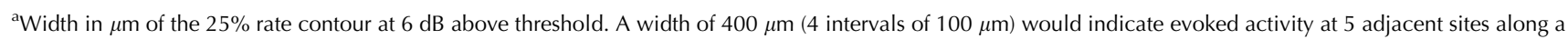
16 -site recording probe.

the frequency that gave a detectable response at the lowest stimulus level. In some cases, offline analysis revealed responses at the lowest stimulus levels tested. In those cases, threshold was designated as the lowest tested level and BF was defined as the frequency that produced the maximum spike count at that level. Spike counts were normalized at each recording site according to the 95th percentile of the average spike counts computed within one wideband stimulus type (a wideband noise of acoustic stimulation or electric activation with bipolar electrodes placed on the round window and oval window). By normalizing in this manner, our results emphasize stimulus-driven changes in activity rather than differences in absolute spike counts across channels. Acoustic STCs were constructed by summing all spikes for the interval beginning at stimulus onset and continuing for the duration of the stimulus. Electrical STCs were constructed by summing all spikes for the interval 5-40 ms following the electrical pulse. The initial 5-ms interval was ignored to avoid including electrical artifacts as part of the response. Typical minimum first spike latencies of ICC neurons to electrical stimulation are 5$5.5 \mathrm{~ms}$. A response focus for each STC was defined as the ICC recording site that produced driven activity at the lowest threshold. The STC-widths were calculated as the width of the $25 \%$ contour at $20 \mathrm{~dB}$ above its threshold for acoustic stimuli (see Fig. 1) and at $6 \mathrm{~dB}$ above threshold for electrical stimuli. Mean STC widths are tabulated in Tables 1 and 2.

\section{RESULTS}

We begin the description of our results by addressing the stability of response waveforms recorded from the ICC of the guinea pig using the multichannel probes. We then briefly summarize the spatial and temporal distribution of neural activity evoked in the IC by acoustic stimulation. Next, we compare acoustic evoked activity patterns with patterns evoked by intracochlear electric stimuli in the same animals after that cochlea had been deafened. Finally, we compare the spatial and temporal patterns of activity evoked by different electrodes and/or different configurations of the same electrode in these same animals.

\section{Response stability and response waveforms}

Stable single-neuron and multineuronal activity patterns were recorded from all animals. The stability of these patterns was confirmed first by comparing acoustic responses and BFs recorded immediately after the probe was fixed in place with those recorded after the animal had been rotated to provide access to the contralateral cochlea. The frequency response areas (FRAs) and BFs of these recordings invariably 
Recording Site 9

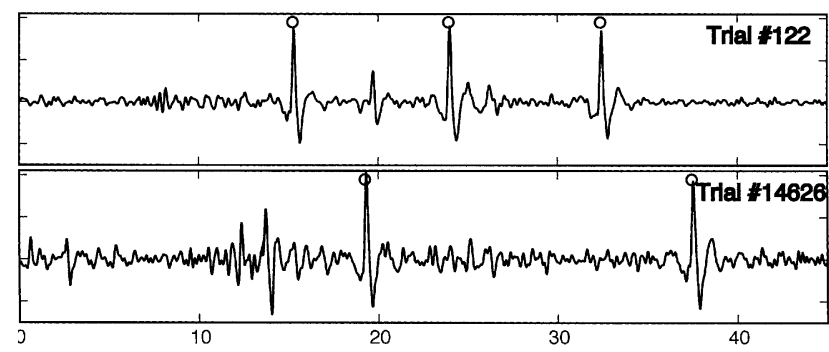

Recording Site 12
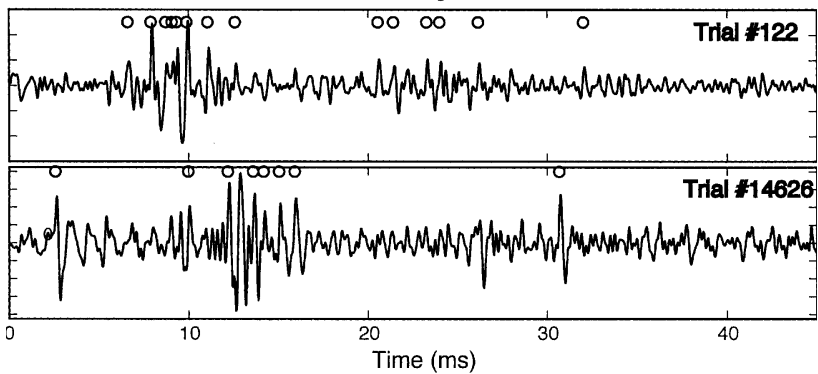

FIG. 2. Neural waveforms from GP26 illustrating activity evoked by an electrical pulse presented at time $=0$. Upper two rows show recordings from a recording site $0.8 \mathrm{~mm}$ (site 9) from the most superficial site. Lower two rows show recordings from a recording site $1.1 \mathrm{~mm}$ (site 12) from the most superficial site. The trial from which the data were obtained is indicated at the top right of each panel. The top panel of each pair of panels was obtained early within an experiment while the lower of each pair of panels was obtained several hours later in the same experiment. The example shown in the top two panels shows an isolated single unit while the lower example shows a multineuronal cluster. In most cases, multineuronal clusters were observed. Circles indicate spikes accepted by the spike-sorting software.

showed no significant differences, indicating that there were no gross movements of the recording probes. Second, in all animals, neural response waveforms recorded at selected sites and evoked by electric stimulation early in the experiments were compared with those recorded at the same sites several hours later in the experiment. Figure 2 illustrates four neural waveforms, $45 \mathrm{~ms}$ in duration, representing neural activity recorded in the ICC of one animal at two probe sites: site 9 (top pair) and site 12 (bottom pair) of one fixed recording probe. The top recording in each pair was recorded simultaneously early in the experiment (trial 122) and the bottom recording in each pair was recorded simultaneously approximately $12 \mathrm{~h}$ later in the experiment (trial 14696). The activity observed in each recording was evoked by an intracochlear electric pulse that was presented at the beginning of each trace. Although not visible in these examples, electric artifacts often were present at the onset of recorded traces. Artifacts always were confined to the first $1-3 \mathrm{~ms}$ of the recordings, however, so they could be distinguished easily from ICC neuronal spikes, which had a poststimulus latency of $>5 \mathrm{~ms}$.

The circles above the spike waveforms indicate waveforms that were recognized as spikes by our spike-sorting algorithm. By comparing activity recorded at site 9 with that recorded at site 12, one can see that the quality of recordings varied among sites on a single probe. In this case, recordings at site 9 are representative of sites at which relatively good isolation of single-neuron spike activity was observed. Recordings at site 12 are representative of sites where relatively poor isolation among neurons was observed. Recordings such as those at site 12 are termed multineuronal or cluster recordings, although clear single neurons are discernable. The quality of recording in these examples is representative of the range of recording quality at some $95 \%$ of recording sites. At the remaining $5 \%$ of sites, recordings showed no consistent spike activity and were excluded from further analysis. The similarity of the recordings at a given site at times separated by several hours indicates the stability of recording that was typical of these experiments. Finally, although unit responses often were stable over the course of hours, the goals of this experiment did not require that responses from specific neurons be recorded throughout the course of all stimulus sets; only coarse stability (no gross change in $\mathrm{BF}$ ) was needed.

\section{Responses to acoustic stimulation}

Each experiment began with stimulation of the contralateral cochlea with a series of acoustic tones that varied across a wide range of frequencies and intensities. The responses to these tones at each site yielded a FRA and an associated BF. Representative FRAs recorded with one probe are illustrated in Figure 3. The excitatory regions of all these FRAs have the V shape that is typical for ICC neurons that we have recorded under ketamine anesthesia. The FRAs are arranged sequentially according to their position along the probe (ICC depth) with the FRA from the most superficial probe site (upper left panel) to the deepest probe site (lower right). The estimated $\mathrm{BF}$ at successive sites shifted systematically from $2 \mathrm{kHz}$ at the most superficial site to $28 \mathrm{kHz}$ at the deepest site.

The progressive change in BF as a function of ICC depth (site location) was consistent across animals. Figure 4 illustrates a plot of BF vs. ICC depth for 9 guinea pigs. All cases showed a similar increase in $\mathrm{BF}$ with increasing recording depth. The median slope was $453.8 \mu \mathrm{m} /$ oct and the range was $380-716 \mu \mathrm{m} /$ oct. Previous studies in cats (Schreiner and Langner 1998; Snyder et al. 1990, 2000) and bats (Fuzessery 1994) using conventional single-electrode recording tech- 


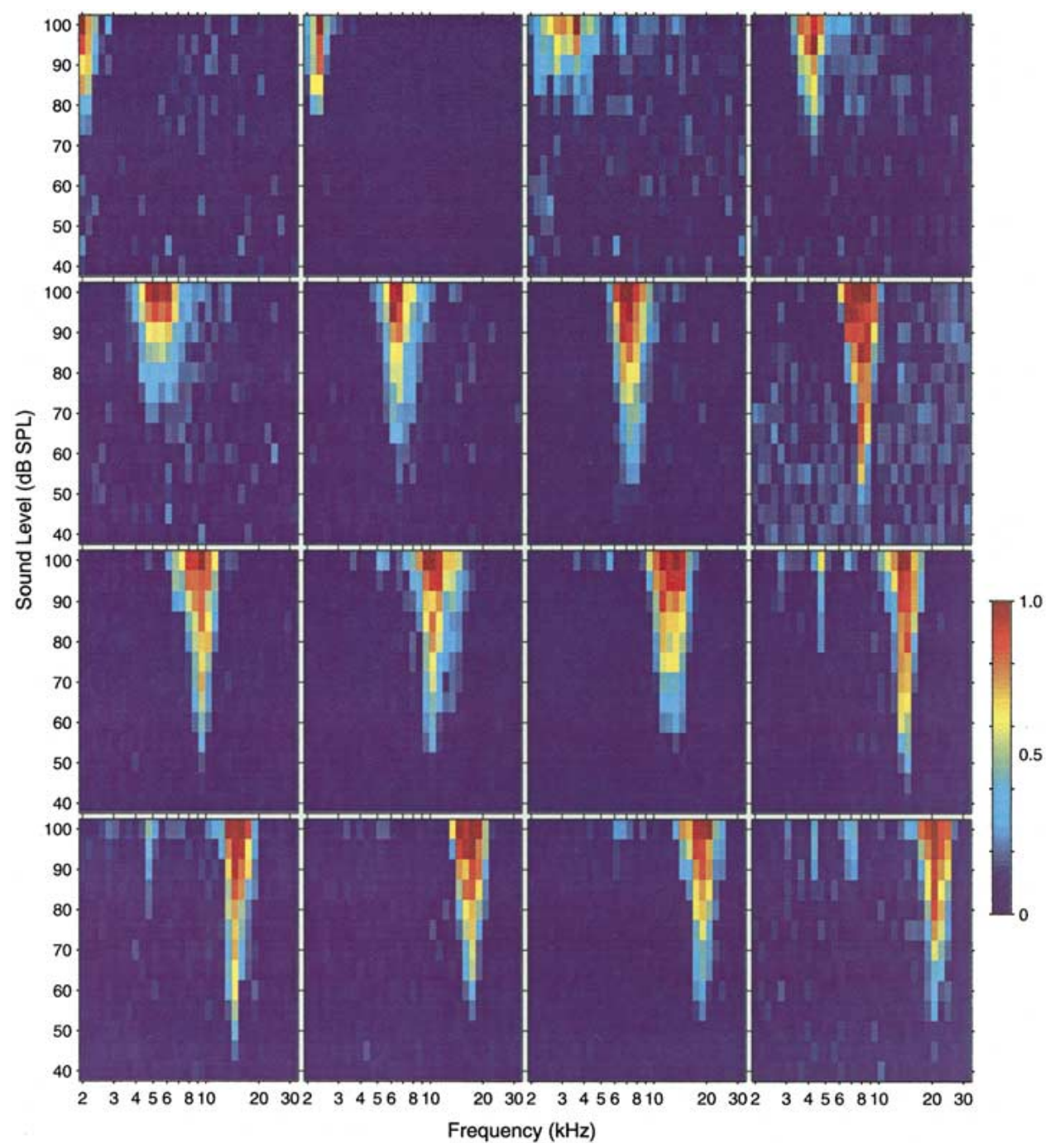

FIG. 3. Acoustic frequency response areas recorded with a 16channel probe in the ICC of a guinea pig. Each of the 16 panels represents measurements from one probe site. In each panel, the abscissa is frequency $(3-32 \mathrm{kHz})$ and ordinate is stimulus intensity (in $\mathrm{dB} \mathrm{SPL}$ ). The normalized response rates are represented in

niques have shown similarly reproducible progressions of $\mathrm{BF}$ as a function of penetration depth.

Figure 3 shows responses at single recording sites as a function of tone frequency and level, but similar responses cannot be routinely obtained using electrical stimulation. Comparisons between acoustic and electric responses are facilitated by representations that show responses to a particular stimulus measured simultaneously at 16 sites through the depth of the ICC. Figure 5 shows four acoustically elicited STCs, which collapse responses across the time dimension and show changes in the spatial spread of excitation across the depth of the ICC as a function of a series of stimulus levels. Each STC shows mean spike rates as a function of depth (site location) and stimulus level. Spike rates are represented by isorate contours, 10\%- pseudocolor with dark blue equal to spontaneous activity and dark red equal to maximum driven response. Response areas from sites distributed sequentially along the probe from most superficial to deepest are distributed from left to right and from top to bottom. GP24.

$90 \%$ of maximum rate at each location in $10 \%$ steps. The leftmost STC illustrates the response to broadband noise, which activated most sites at almost all stimulus levels greater than $40 \mathrm{~dB}$ SPL. At low levels, the contours are nearly vertical stripes showing little site selectivity, i.e., approximately the same threshold and rate response at all locations. At higher levels, the activity pattern is irregular with patches of maximum rate responses at idiosyncratic locations within the depth/intensity matrix. The remaining three toneevoked STCs illustrate activity patterns evoked by 5-, $10-$, and $20-\mathrm{kHz}$ tones. The contours for each tone consist of a series of nested Vs indicating significant excitation selectivity at all stimulus intensities. The mean STC width, averaged across all animals and tested frequencies, was $382 \mu \mathrm{m}$. Thus, at $20 \mathrm{~dB}$ above 


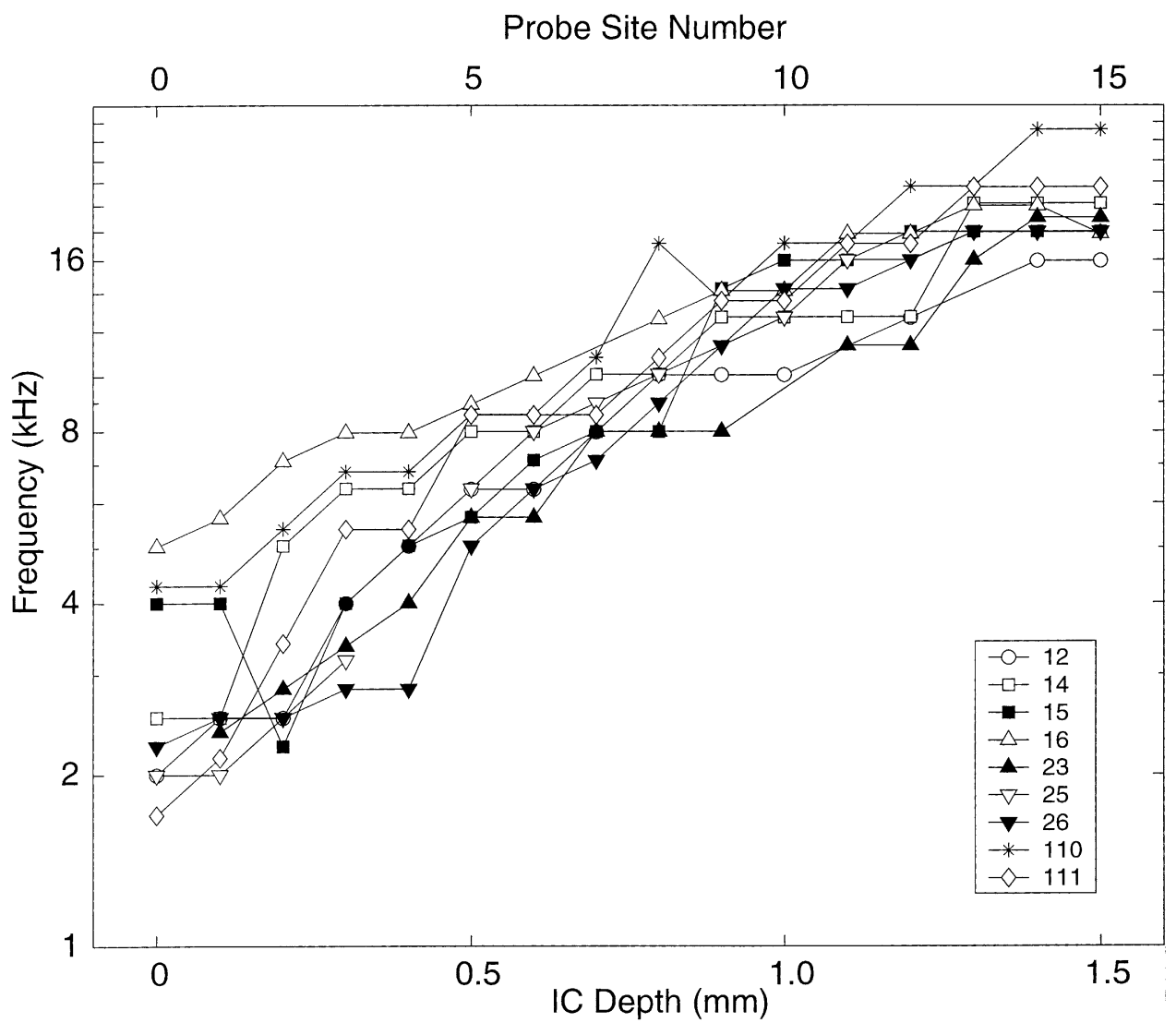

FIG. 4. Frequency gradients recorded from 9 multichannel recording probes in 9 animals identified by the key.

threshold, most tones activated neurons spread across approximately 5 probe sites. In contrast, noise at 20 $\mathrm{dB}$ above threshold activated neurons distributed further than the total extent of the recording probes $(>1500 \mu \mathrm{m})$.

\section{Intracochlear electric stimulation}

We employed two types of electrode for intracochlear electric stimulation: VP ball electrodes and a multichannel-banded electrode array. The advantage of the VP electrodes was that they could be placed in known positions relative to specific cochlear structures. Using the VP electrodes, we could test within an individual animal the influence of radial vs. longitudinal orientation of electrode pairs, the influence of proximity to the spiral ganglion (see Figs. 8 and 10), and the influence of interelectrode separation of a bipolar pair. Moreover, their positions could be documented in detail. The disadvantage of the VP electrodes was that they are unlike any device that presently is in clinical use. For that reason, we also tested a multichannel-banded electrode array that is similar to the Nucleus 22 device from the Cochlear Corporation. Using the banded electrode array, we could compare responses to various monopolar, bipolar, and tripolar stimulus configurations that are possible in clinical devices. Also, we could measure the cochleotopic progression of ICC responses to stimuli at multiple uniform longitudinal increments along the cochlear spiral.

Visually placed ball electrodes. Figure 6 shows a ball electrode pair in place in a cochlea; left and right panels show, respectively, a digital image acquired through the operating microscope and a drawing of that image. In this preparation, the lateral wall of the bulla has been removed exposing the lateral surface of the cochlea. The basal end of the cochlea is to the left and the apical end is to the right. All four cochlear turns (I, II, III, and IV) can be seen and are labeled in the drawing on the right. The lateral wall of the basal end of the first cochlear turn has been removed by enlarging the round window and exposing the basilar membrane of the hook and basal end of the first turn. The electrodes were placed in a radial orientation on the osseous spiral lamina, with one electrode (A) placed on the habenula and the other (B) placed over Rosenthal's canal.

Effects of longitudinal position. Figure 7 shows the IC responses evoked by VP electrodes placed on the osseous spiral lamina at successively more basal (higher frequency) locations along the length of the 


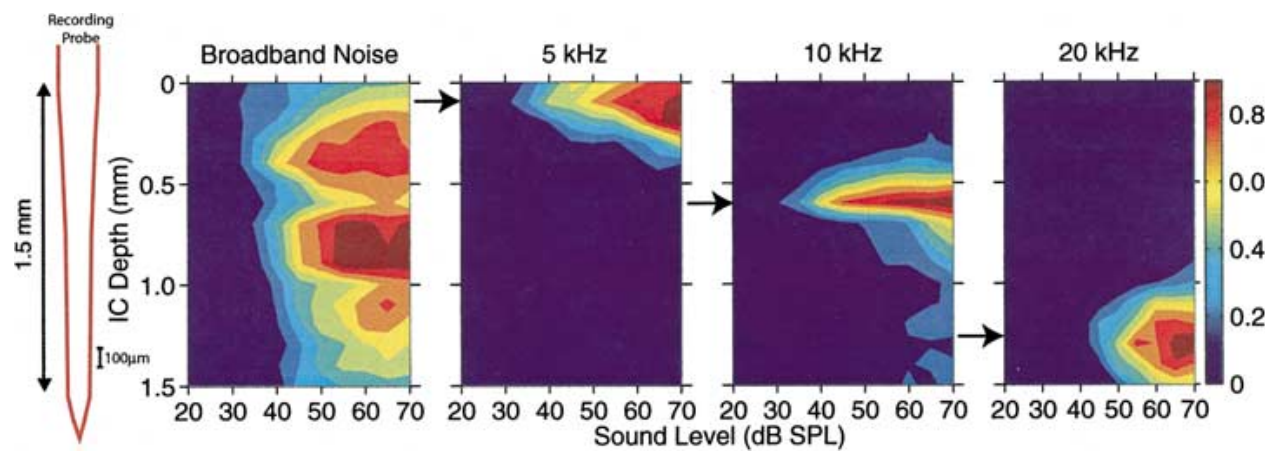

FIG. 5. Spatial tuning curves (STCs) evoked by broadband noise and tone bursts at 5, 10, and $20 \mathrm{kHz}$. In each STC, the vertical dimension is ICC depth (i.e., distance along the recording probe) and the horizontal dimension shows stimulus intensity. The colors represent normalized spike rates indicated by the pseudocolor scale shown at the right. In the noise STC, activity was broadly distributed even at low stimulus levels. In each tone-evoked STC, activity was spatially restricted at near-threshold levels and spread across the cochleotopic axis as tone intensity increased, In most cases, the region of lowest threshold corresponded to the response focus, i.e., region of maximum response. The response focus shifted ventrally as the stimulus frequency increased. GP16.

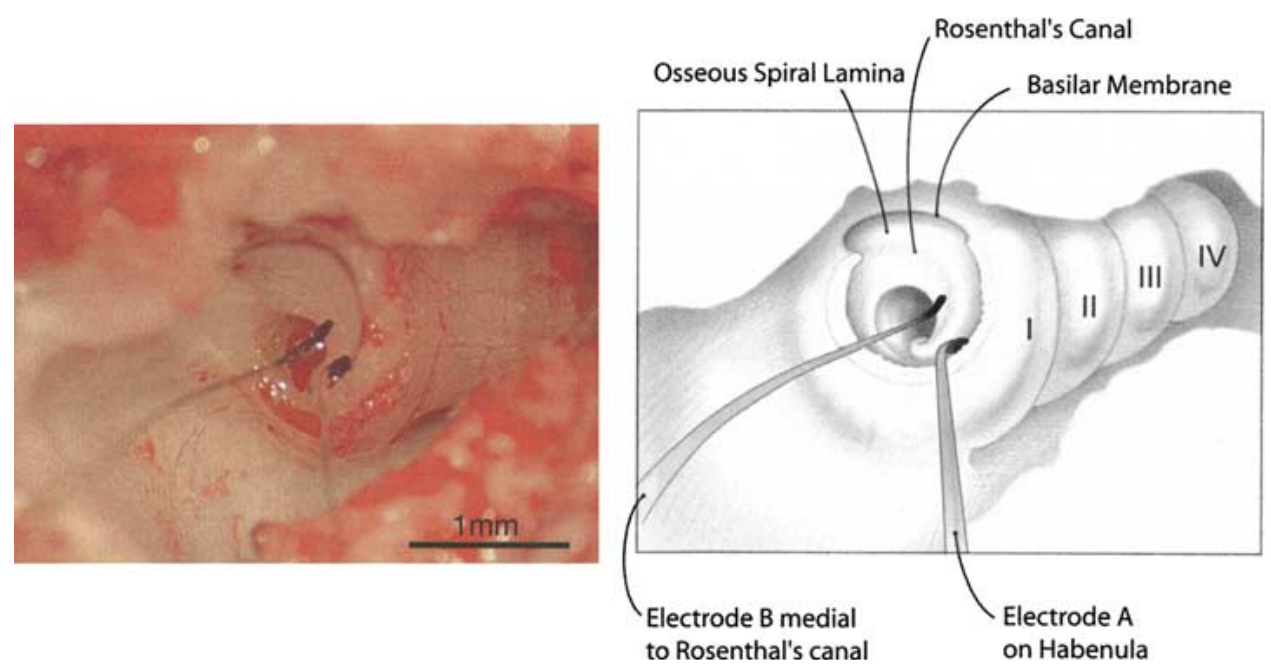

FIG. 6. Photographic image (left) and drawing (right) of two visually placed, radial bipolar electrodes. The lateral wall of the bulla and cochlear basal turn has been removed so that the osseous spiral lamina can be seen in the hook and initial basal turn regions of the cochlea. The four turns of the guinea pig cochlea are indicated by the Roman numerals I-IV. GP23.

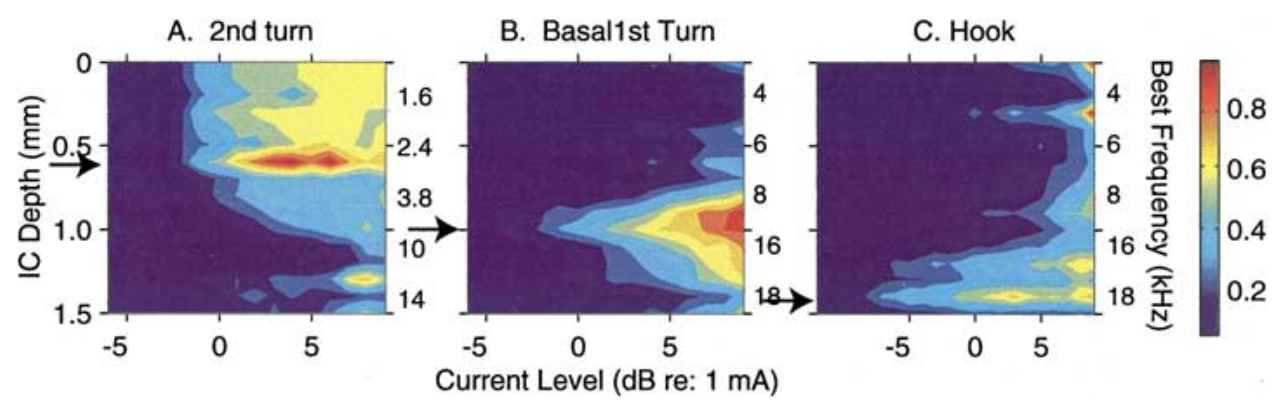

FIG. 7. Spatial tuning curves (STCs) illustrating the spatial distribution of activity evoked by electrical pulses delivered via visually placed (VP) electrodes at three locations within the cochlear spiral. A. VP electrodes in the second turn. GP13. B. VP electrodes in the basal first turn. GP15. C. VP electrodes in the hook region. GP15. Responses were normalized relative oval/round window stimulation. The response focus for each STC is indicated by the black arrows. All stimuli were single biphasic pulses $40 \mu \mathrm{s} /$ phase.

cochlea: second turn (Fig. 7A) of one animal, and basal first turn (Fig. 7B) and middle of hook region (Fig. 7C) of another. As expected from the cochleo- topic organization of the ICC, successively more basal electrode placements resulted in activity patterns focused at successively more ventral (higher frequency) 


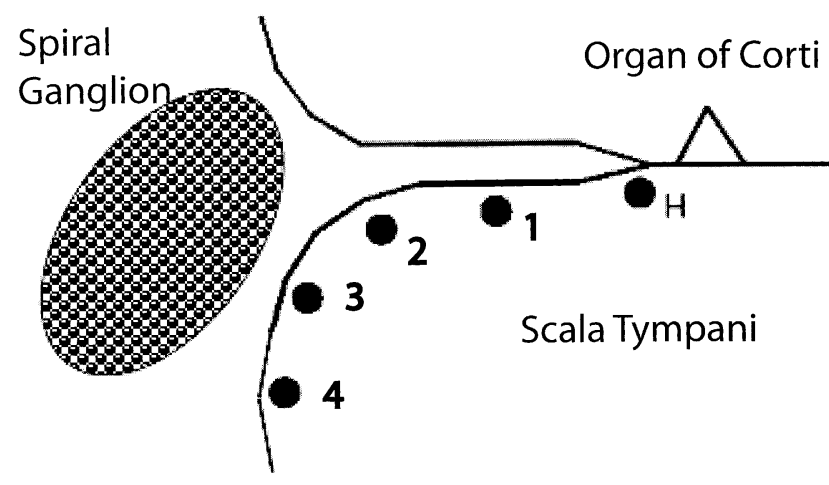

FIG. 8. Diagram of five radial electrode placements used with VP radial electrodes to test the effects of different radial electrode placements in the basal cochlea on threshold and spatial spread of evoked activity in the ICC.

ICC locations. Stimulation of the second turn produced activation that was focused at an ICC recording site that was located $0.6 \mathrm{~mm}$ (site 7) along the recording probe. That site, when tested with tones prior to deafening this animal, was tuned to relatively low frequencies, approximately $2.8 \mathrm{kHz}$. Stimulation in the basal first turn (Fig. 7B) of another animal produced an activation pattern with a response focus centered at $1.0 \mathrm{~mm}$ (site 11) along the probe. This site was tuned to frequencies around $10 \mathrm{kHz}$, corresponding to the estimated $\mathrm{BF}$ of the auditory nerve fibers (ANFs) innervating this higher-frequency region of the cochlea. When the electrode was moved still further basally into the hook region (Fig. 7C), it produced an activation pattern that had a response focus and maximum response centered at $1.4 \mathrm{~mm}$ (site 15), which was tuned to frequencies around 18 $\mathrm{kHz}$. Thus, shifting the longitudinal site of intracochlear stimulation with VP electrodes produced spatially restricted activation at appropriate regions of the tonotopically organized ICC.

In the case shown in Figure 7A, the spread of ICC activation by stimulation at the most apical site was considerably broader than that resulting from more basal stimulation. At least two factors could have contributed to the greater breadth of activation from this apical site. First, the size of the scala tympani relative to the size of the VP balls in the second turn required that the VP electrodes be oriented longitudinally, straddling the modiolus, whereas more basal placements could be oriented closer to a true radial configuration. We show in the next section that longitudinal orientations result in broader spread of activation. Second, while the more apical electrode placement produced maximum activation of neurons tuned to approximately $3 \mathrm{kHz}$, at higher stimulus levels these electrodes also activated IC neurons that were tuned to lower frequencies. This "ectopic" activation could have been due to activation of lower- frequency ANFs that must pass close to the secondturn electrodes on their way down the modiolus toward the internal meatus. That is, at higher stimulus levels, current could invade the modiolus and activate fibers that innervate more apical locations. A similar activation pattern was predicted by a three-dimensional spiral model of intracochlear current spread for similar longitudinal electrodes (see Fig. 10B in Briaire and Frijns 2000). The activation of apical fibers of passage does not occur following activation of the basal electrodes since the diameter of the scala tympani in the first turn is approximately twice that of the second turn. VP electrode pairs could be placed in a radial orientation there, resulting in a more restricted spread of current that was less likely to involve fibers passing from more apical sites.

Comparison of the STCs in Figure 5 with those in Figure 7B,C indicates that the spatial selectivity of the electric-pulse-evoked STCs using radially placed VP bipolar electrodes was comparable to that observed for STCs evoked by acoustic tones. The mean width of STCs for radial bipolar VP pairs was $429 \mu \mathrm{m}$ when stimulated at $6 \mathrm{~dB}$ above threshold, whereas the mean width for tone-evoked STCs at $20 \mathrm{~dB}$ above threshold was $382 \mu \mathrm{m}$. Shifting the locations of stimulating electrodes along the cochlear spiral shifted the regions of minimum threshold and maximum activation within the ICC in a way that is consistent with the tonotopic connections between the cochlea and ICC.

VP electrodes: effects of radial position and electrode separation. The effects of systematically varying radial location and separation between VP bipolar electrodes on STCs were examined using five electrode positions distributed across the radial dimension of the first cochlear turn (Fig. 8). In these experiments, one contact was placed either on the habenula $(\mathrm{H})$ or on the osseous spiral lamina at one of four locations distributed between the proximal modiolus (location 4, Fig. 8) and the distal osseous spiral lamina (location 1, Fig. 8). The other contact was placed at one of the other locations.

The spread of excitation evoked by activation of radial VP electrodes at these locations was examined in three animals. Examples of the STCs evoked by activation of one electrode pair at four radial separations $(4, \mathrm{H} ; 3, \mathrm{H} ; 2, \mathrm{H}$; and $1, \mathrm{H})$ in two animals are illustrated in Figure 9. These combinations were chosen to represent the responses evoked by both closely $(1, \mathrm{H})$ and widely separated $(4, \mathrm{H})$ electrode pairs. The STCs in the upper row summarize the spread of activation for these electrode separations over a broad range of stimulus intensities. For all four separations, activation spread increased monotonically as stimulus intensity increased, but the regions of maximum evoked activity remained relatively restricted except at the highest stimulus levels. Mini- 

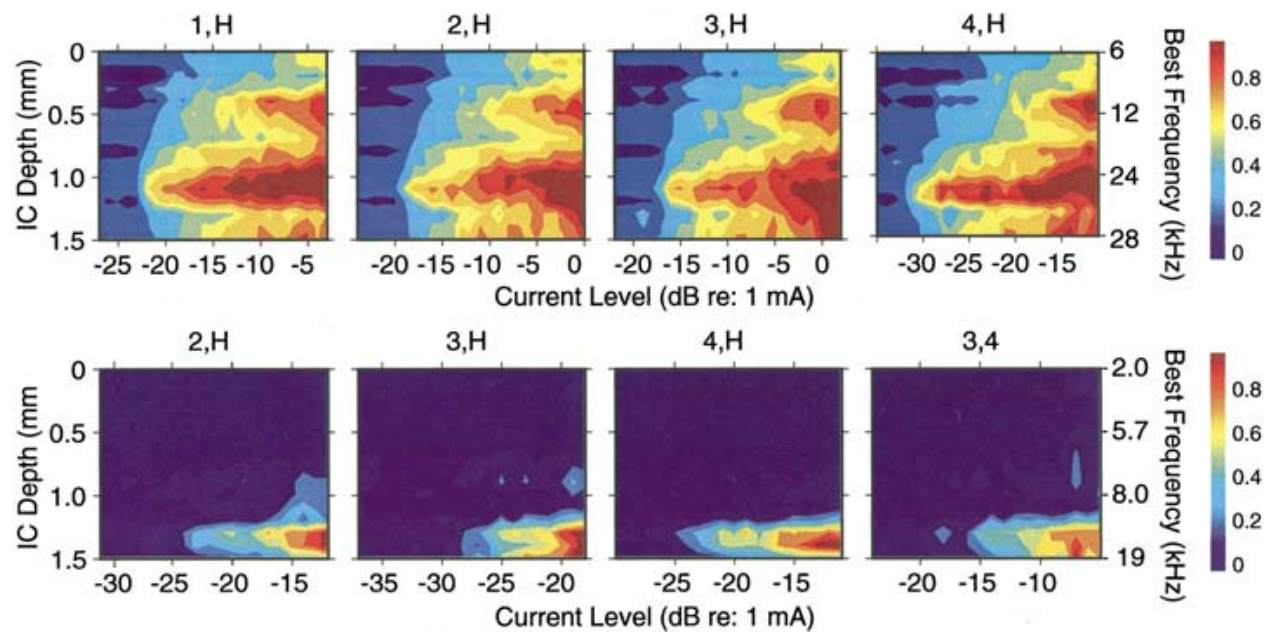

FIG. 9. Upper row: Spatial tuning curves (STCs) obtained using a pair of radial bipolar VP electrodes, one over the habenula perforata (H) and the other at locations successively further from it (see placements 1, 2, 3, or 4 in Fig. 8). GP29. Lower row: STCs evoked by

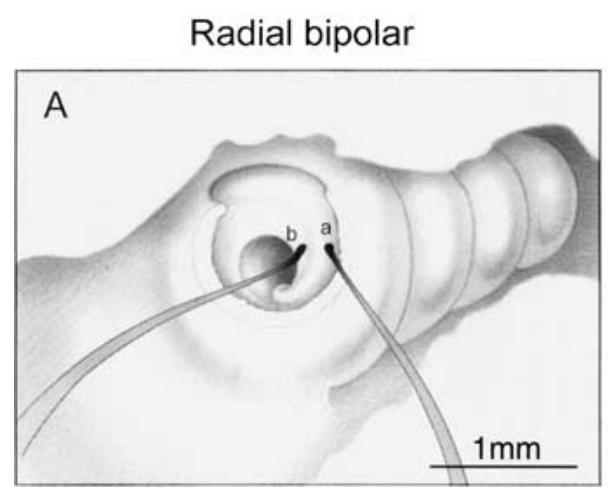

the electrode positions indicated above each panel (see Fig. 8). The $\mathrm{VP}$ electrodes in the far right column were positioned at sites 3 and 4, straddling the spiral ganglion. GP23.

FIG. 10. Drawings of VP electrodes in the cochlea of a guinea pig. A. Visually placed ball electrode pair oriented radially at $\mathrm{H}$ and 3 on the osseous spiral lamina. B. VP electrodes placed longitudinally spanning the boundary of the hook and first turn. Electrode is placed on the osseous spiral lamina over or just distal to Rosenthal's canal and the spiral ganglion (location 3, Fig. 8). GP23.

mum threshold currents at all sites tended to be lower for more widely separated pairs $(4, \mathrm{H})$ than they were for closely spaced pairs $(2, \mathrm{H}$ and $1, \mathrm{H})$, but this was not always observed $(3, \mathrm{H})$.

The STCs in the bottom row of Figure 9 illustrate another set of VP electrodes in a second animal. In this case, evoked activity is restricted at all stimulus intensities to a few sites with BFs centered at $15 \mathrm{kHz}$. In these recordings, the spread of both threshold and maximum activity is highly restricted at all stimulus levels. These STCs are comparable to those observed with acoustic stimulation using a $20-\mathrm{kHz}$ acoustic tone (see Fig. 4). The STCs in Figure 9 are representative of those evoked using radial VP electrode pairs. The radial position of radial electrodes and the magnitude of their separation appear to have little effect on the spread of activation across the IC, although they can have an effect on the minimum threshold of the responses. The STCs evoked by activation of $1, \mathrm{H}$

\section{ICC STCs}

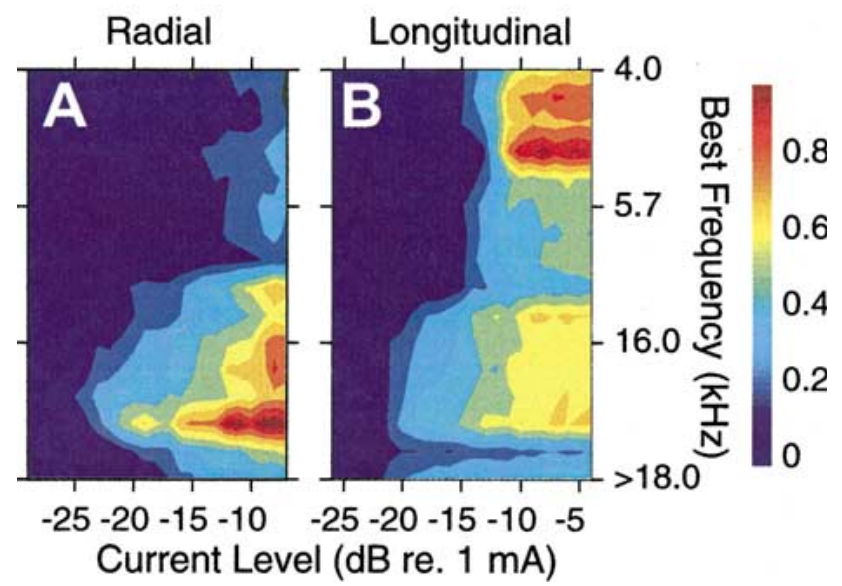

FIG. 11. A,B. STCs evoked by the same electrodes seen in Figure 10. GP23. 


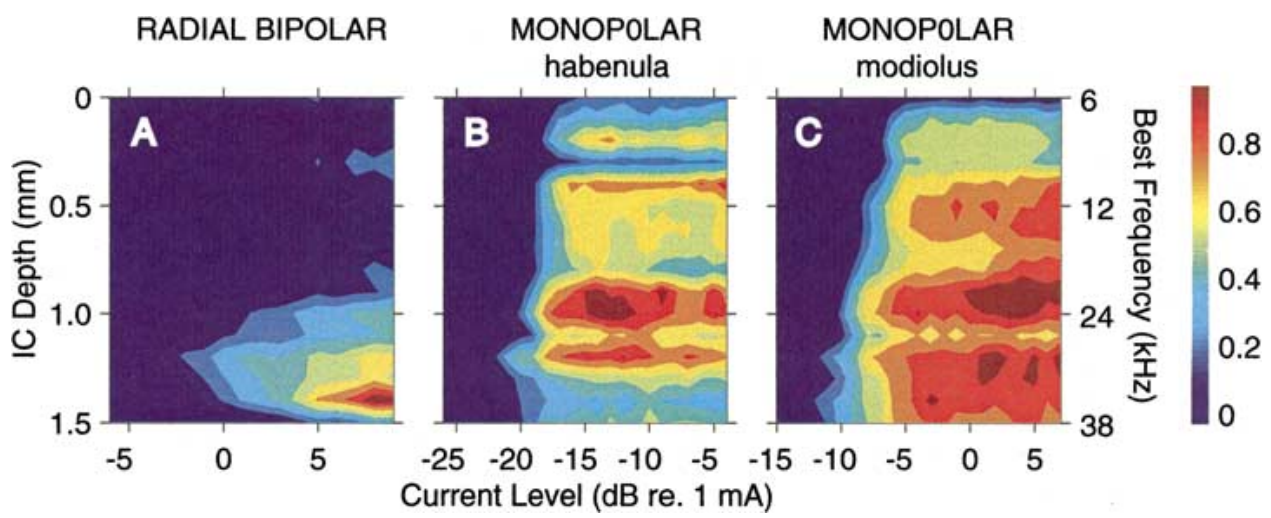

FIG. 12. Spatial tuning curves (STC) evoked by pulses delivered to the basal turn of guinea pig GP15. A. STC evoked by stimulation with bipolar radial VP electrodes. B. STC evoked by monopolar stimulation with pulses delivered via the habenular electrode of the VP pair used in A. C. STC evoked by monopolar stimulation with pulses delivered via the modiolar electrode used in $\mathbf{A}$.

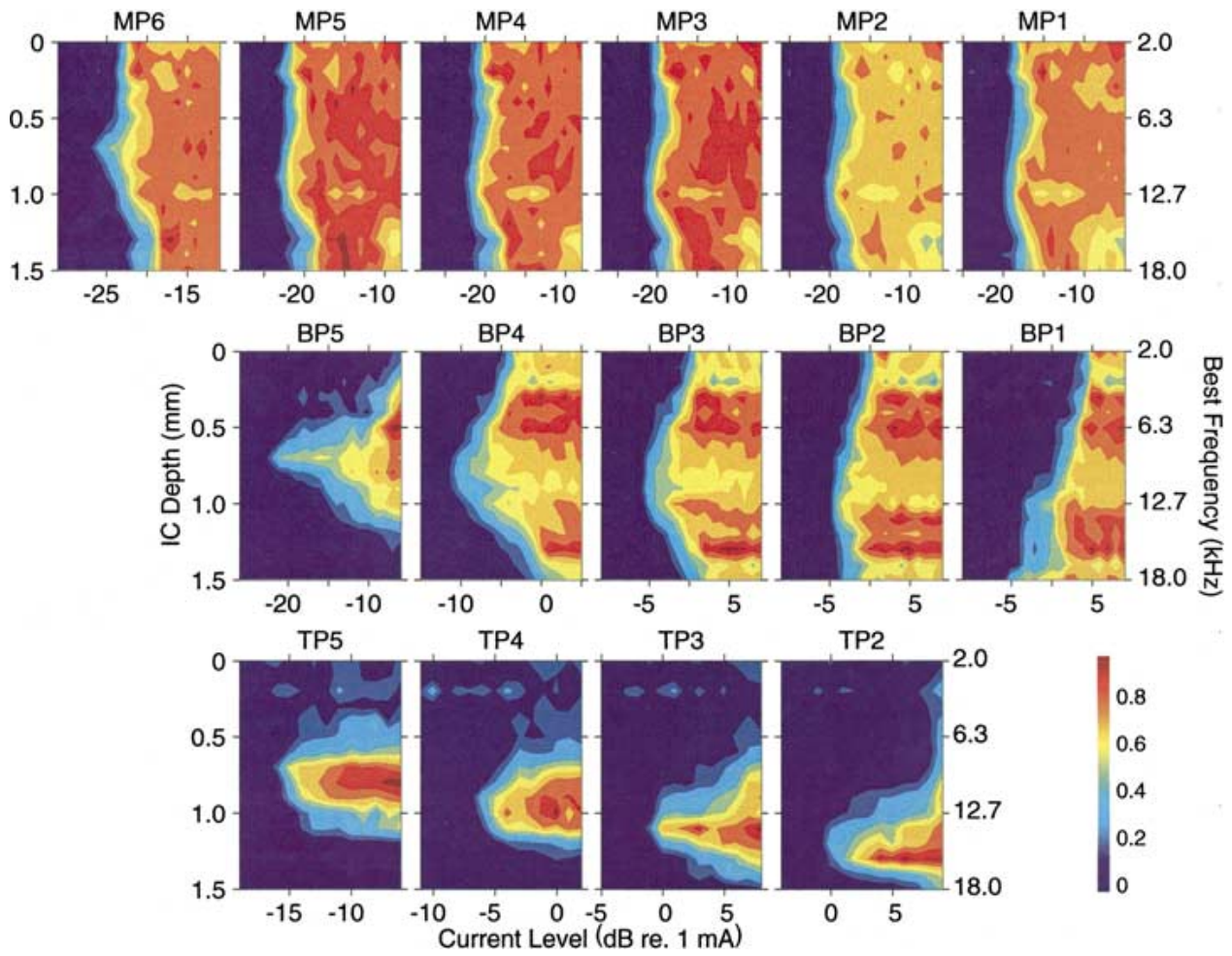

FIG. 13. Spatial tuning curves (STCs) evoked by activation of a banded electrode. Activation of all bands across the entire recording array at current levels at least $1-2 \mathrm{~dB}$ below to at least $2 \mathrm{~dB}$ above threshold are illustrated. Upper row: Each band activated as a monopole (MP) electrode. MP6 is the most apical band; MP1 is the most basal band. Middle row: Each adjacent pair of bands activated

(upper row) and 2,H (lower row), which are closely spaced pairs located near the habenula, are similar to those evoked by $4, \mathrm{H}$, the most widely spaced pair. More widely separated electrode pairs tended to have lower thresholds, whereas more closely spaced electrodes tended to have higher thresholds. These results using radially oriented VP electrodes lead us to as bipoles, BP. BP1 $=$ activation of bands 1 and 2 ; BP5 $=$ activation of bands 5 and 6 , etc. Bottom row: Adjacent triads of electrodes activated as tripoles, center band acting as active and adjacent bands acting as return electrodes. TP2 = activation of bands 1, 2, and 3; TP5 = activation of bands 4, 5, and 6. Stimuli were biphasic pulses $160 \mu$ s/phase. GP25.

conclude that, as long as electrodes are in contact with the osseous spiral lamina, radial electrode separation and radial placement relative to the excitable neural elements (unmyelinated or myelinated dendrites, ganglion cell bodies, peripheral axons, etc.) have little effect on the spread of excitation along the cochlear spiral. 
VP electrodes: effects of orientation. In contrast to the small effects of radial bipolar electrode separation and placement on activation spread, the orientation (radial vs. longitudinal) of bipolar electrodes had a strong influence on activation spread. The patterns of ICC activity evoked by VP electrode pairs oriented longitudinally had higher thresholds, broader regions of activation near threshold, and more idiosyncratic locations of maximum activation than did electrode pairs oriented radially in the same animal. One example of these effects was shown earlier when second turn and basal turn STCs were compared (see Fig. 7). Figure 10 illustrates a more systematic approach to the study of electrode orientations. Figure $10 \mathrm{~A}$ shows a radially oriented VP electrode pair (a,b) placed at locations $\mathrm{H}$ (a) and 3 (b) (see Fig. 8) on the osseous spiral lamina at the basal end of the first cochlear turn. Figure 10B shows the same electrode pair oriented longitudinally. Electrode b remained at its original location. Electrode a was moved basally to the middle of the hook region, a location corresponding to that of electrode $b$ but at a higher frequency location. Thus, this longitudinal orientation spanned the boundary between the hook and first turn at locations that overlay the spiral ganglion (corresponding to radial position 3, see Fig. 8).

Figure 11 shows STCs evoked by the radial (A) and longitudinal (B) electrode orientations illustrated in Figure 10. In Figure 11A, the spread of activation for radial pair was somewhat broader than but roughly comparable to those seen for other radial VP electrodes (see Fig. 8). Near threshold, the spread of activation was restricted to little more than two sites, 12 and 13 (depths of 1.1 and $1.2 \mathrm{~mm}$ ). At higher intensities, evoked activity spread only moderately, with the highest response rates ( $>10 \%$ of maximum) centered on site 14 (depth $1.3 \mathrm{~mm}$, corresponding to a $\mathrm{BF}$ of $13 \mathrm{kHz}$ ) and the rates $>30 \%$ of maximum spreading to sites with BFs between 8 and $16 \mathrm{kHz}$. This restricted activation suggests that these radially oriented electrodes continue to activate relatively restricted regions of the auditory nerve array across a range of currents comparable to the dynamic range of a typical cochlear implant user. In Figure 11B, the pattern of activation for the same electrodes in a longitudinal orientation was significantly broader. At low stimulus levels, the near-threshold responses were distributed across sites that were anticipated based on the intracochlear locations of the electrodes and the responses to tones in the measurements made prior to deafening. The response focus was located somewhere between sites 8 and 15, which had BFs between 8 and $16 \mathrm{kHz}$. At higher stimulus levels, the maximum response region shifted to inappropriate locations, sites 2-4, which represented frequencies between 3 and $6 \mathrm{kHz}$. These results are consistent with the suggestion that longitudinally oriented electrodes activate not only nearby auditory nerve dendrites but also activate auditory nerve fibers of passage within the modiolus that innervate more apical cochlear locations (see Fig. 7). The effects of radial vs. longitudinal electrodes' orientations can be summarized by average widths of their STCs. Radially oriented VP electrodes evoked STCs with a mean 6-dB width of $429 \mu \mathrm{m}$, whereas STCs evoked by longitudinally oriented VP electrodes separated by a similar distance had a mean 6 - $\mathrm{dB}$ width of $719 \mu \mathrm{m}$. The longitudinal electrodes activated nearly twice as much of the ICC as the radially oriented balls (Table 2). Off-radial balls, those with both a radial and a longitudinal component to their separation, had a mean STC width of $751 \mu \mathrm{m}$.

VP electrodes: mono- vs. bipolar configurations. In all of the cases illustrated to this point, electric stimuli with VP electrodes were presented in a bipolar configuration in which one electrode was the active electrode and the other electrode was the return. Figure 12A illustrates another such case, showing the spread of activation in the ICC in response to stimulation of a radial bipolar VP electrode pair located in the basal first turn. Figures 12B and C, in contrast, show the activation elicited by either of those VP electrodes stimulated as a monopolar active electrode with a distant contact in a neck muscle serving as the return electrode. Both of the monopolar cases show broad spread of activation in the ICC. At nearthreshold levels, the monopolar activation patterns are centered at high-frequency locations comparable to those at which the bipolar patterns are centered, site 12 ( $\mathrm{BF} \sim 24 \mathrm{kHz}$ ) for the habenular electrode (Fig. 11B) and site $14(\mathrm{BF} \sim 32 \mathrm{kHz})$ for the modiolar electrode (Fig. 12C). At higher levels, however, activity was broadly distributed and patchy. Although the thresholds were 10-15 $\mathrm{dB}$ lower for monopolar stimulation and the response focus of each monopolar pattern was approximately the same as that seen following bipolar stimulation, the activation contours at midrange intensities were nearly vertical stripes stretching across all of the probe sites. The mean STC width at a level $6 \mathrm{~dB}$ above threshold for monopolar stimulation using VP balls was $>1500 \mu \mathrm{m}$, i.e., wider than that of any bipolar configuration and comparable to that for monopolar stimulation with banded electrode arrays (Table 2).

Banded intracochlear electrodes. Figure 13 illustrates representative examples of STCs evoked by activation of a banded electrode that was inserted into the scala tympani through a cochleostomy in the lateral wall of the cochlea just lateral to the round window. STCs evoked by stimulation of this electrode in three configurations are illustrated. The top row illustrates STCs evoked by monopolar stimulation, i.e., activa- 
tion of each band using a remote extracochlear electrode as the return. The middle row illustrates STCs evoked by bipolar stimulation, i.e., two adjacent bands activated as longitudinal pairs. The bottom row illustrates STCs evoked by longitudinal tripolar stimulation, i.e., activation of one band with two flanking adjacent bands acting as the return electrodes.

Monopolar activation of any of the bands produced a broad, idiosyncratic activation pattern in the ICC similar to those seen with VP ball electrodes stimulated as monopoles. The isorate contours are stripes that stretch the entire length of the recording probe. At near-threshold levels there was a tendency for the response focus to shift ventrally as the more basally located bands were activated (compare MP6, the most apical band with a best location at $700 \mu \mathrm{m}$, to MP1 and 2 , the most basal bands with best locations at 1000 $\mu \mathrm{m})$. Monopolar stimulation with the banded electrode consistently produced broad excitation, and appeared to signal the place of cochlear activation only coarsely. The mean $6 \mathrm{~dB}$ widths of the monopolar banded STCs were greater than $1500 \mu \mathrm{m}$, i.e., greater than the entire span of the recording electrode.

Bipolar activation of these same bands usually produced more restricted excitation patterns. The mean STC width of the banded bipolar pairs (684 $\mu \mathrm{m})$ was comparable to that seen with longitudinal VP bipoles, although their threshold and selectivity were highly variable. Some of this variability could be directly related to the intracochlear location of the bands and the size of the scala tympani relative to the bands. Bipolar activation of the most apically located band pair (BP5, i.e., activation of bands 5 and 6) produced low threshold excitation and restricted excitation patterns. In contrast, bipolar activation of more basally located pairs, for example BP1 (i.e., activation of bands 1 and 2 the most basal bands), produced excitation with higher thresholds and selectivity that was broader and more comparable to that seen with monopolar stimulation. This difference in threshold and activation selectivity is assumed to be due to the difference in the cross-sectional area of the scala tympani at the different intracochlear locations of the bands. The scala tympani narrows progressively from base to apex and is relatively narrow in the upper basal and second turns. For example, the apical bands ( 5 and 6 ) nearly fill the scala at these locations. They are forced to lie close to the modiolus and the spiral ganglion that it contains. At more basal locations, the scala tympani gradually expands to more than twice its second-turn diameter and broadens even further in the hook region. At these more basal locations, the bands can reside relatively far from the modiolus and its neural elements. This greater distance presumably accounts for the higher thresholds and poorer excitation selectivity produced by activation of the more basal bands (especially 1, 2, and 3 ).

Tripolar activation (activation of one band with the adjacent bands on either side each returning half the current) produced highly selective excitation but at high thresholds. Tripolar thresholds were comparable to those seen with the most selective longitudinal bipolar stimulation, but they were 10-20 dB higher than those seen with monopolar stimulation. As with bipolar stimulation, thresholds for tripolar contacts located at the most apical location were usually lower than those located more basally. This result is consistent with a higher percentage of current shunting between electrodes in the larger basal scala. However, the excitation patterns evoked by tripolar stimulation were much more consistent among electrodes than those seen with bipolar or monopolar stimulation. STCs evoked by tripolar stimulation were among the most restricted we observed. They were comparable to those seen with radial VP electrodes. Moreover, spread of excitation did not vary systematically with location along the cochlear spiral. Thus, the STCs for the 4 tripoles in Figure 13 were highly restricted and have comparable 6$\mathrm{dB}$ widths. The average $6-\mathrm{dB}$ width for all tripoles was $548 \mu \mathrm{m}$ (Table 2), approximately that seen for radial VP balls. Moreover, the response focus shifted systematically from more superficial to deeper ICC locations as tripolar cochlear stimulation sites were shifted from more apical to more basal locations. The STC widths and shifts in response focus indicate that activation of each of these tripoles excites a different, segregated, and topographically appropriate sector of the auditory nerve array.

\section{DISCUSSION}

The results of these experiments lead to several conclusions: First, electric excitation of restricted sectors of the auditory nerve array at different spiral locations produces cochleotopically appropriate activity patterns in the ICC. Activation of electrodes in basal regions of the cochlea preferentially evoked neural activity in the high-frequency ICC regions, and activation of electrodes located in more apical regions preferentially evoked activity in the lower-frequency ICC regions. The cochleotopic specificity of electric stimulation is expected since several previous studies have shown similar effects in both the IC (Snyder et al. 1990, 1991; Leake et al. 1991, 1995, 2000; Shepherd et al. 1999; Rebscher et al. 2001) and auditory cortex (Woolsey and Walzl 1943; Taniguchi et al. 1997; Klinke et al. 1999; Raggio and Schreiner 1999; Bierer and Middlebrooks 2002; Middlebrooks and Bierer 2002). 
Second conclusion, the orientation of bipolar electrode pairs strongly affects the spatial selectivity of their evoked activity patterns. Radially oriented electrode pairs produced more restricted ICC activation patterns (and by inference more restricted activation of the auditory nerve array) than did longitudinal pairs. This conclusion is consistent with the results of studies of the influence of electrode orientation on physiological activation selectivity in the auditory nerve of hearing cats (Van den Honert and Stypulkowski 1987). This study reported that radial bipolar electrodes activated the auditory nerve highly selectively, whereas longitudinal bipolar electrodes produced much broader activation.

Third conclusion, the radial separation within a bipolar electrode pair has little effect on the spatial selectivity of the resulting activity patterns. Activity patterns evoked by VP bipolar pairs that were located peripherally (i.e., closer to the habenula) and close together were no more selective than those located centrally (i.e., closer to Rosenthal's canal) and/or separated as widely as possible. Moreover, although electrode separation could have a strong effect on the minimum threshold, this effect was not seen consistently. These conclusions conflict with the modeling results reported by Frijns and colleagues (1996), which were generated using the boundary element method to compute the spread of current in a rotationally symmetric model of the guinea pig cochlea. When those investigators computed the spread of current for symmetric biphasic pulses, they found dramatic differences between electrode pairs based on both their spatial separations and their radial placements. Modeled current spread also depended on the proximity of electrodes to the osseous spiral lamina, although that parameter was not tested in the present study. In the modeling results, widely separated electrode pairs produced excitation profiles with high thresholds, relatively poor selectivity, relatively narrow dynamic ranges, and relatively large ectopic regions of excitation. Model electrode pairs spaced close together, especially those located over the habenula, displayed lower thresholds, extremely narrow spatial tuning, and wide dynamic ranges $(>20$ $\mathrm{dB})$. Our experimental data showed none of these effects. Radial electrodes with different separations between contacts evoked activity patterns with comparable spatial distributions within an individual animal, although these patterns differed substantially between animals (compare the STCs in the top row with those in the bottom row of Fig. 9). Within an animal, minimum thresholds varied significantly, but not consistently, as a function of electrode separation. Electrodes placed more closely together tended to have higher minimum thresholds than those that were more widely separated, but not always (as in the upper row of Fig. 9). One hypothesis, which might account for the higher thresholds of more closely spaced electrodes, is that for such electrodes more current can shunt directly between contacts and less can penetrate into the tissue. One might model this effect by adjusting the relative impedances of cochlear tissues and perilymph fluids; however, reconciliation of model predictions with experimental results must await an extensive series of modeling-experiment-remodeling iterations. Such a series is beyond the scope of this first series of experiments.

The fourth conclusion from our results is that when banded or ball electrode pairs are oriented longitudinally (i.e., when they are placed at two sites along the cochlear spiral), electrode separation has a strong and consistent effect on both threshold and spatial selectivity of the evoked activity patterns. Longitudinally oriented bipoles produced less selective activation patterns at lower thresholds than more narrowly spaced longitudinal bipoles. Three experimental studies have examined the effects of electrode separation for longitudinally oriented bipoles. Snyder and colleagues (1990) reported that the 6 - $\mathrm{dB}$ widths of STCs evoked by longitudinal bipoles separated by 4 $\mathrm{mm}$ were twice as broad as those evoked by bipoles separated by $1 \mathrm{~mm}$. However, Rebscher and coworkers (2001) reported that the mean 6-dB widths of STCs for closely spaced and widely separated bipolar electrodes were not significantly different. They suggested several reasons for this result including double minima for widely separated bipoles. Bierer and Middlebrooks (2002) reported that increases in the spatial separation between bands of a modified $\mathrm{Nu}-$ cleus electrode led to "a slight increase" in the width of cortical images. Frijns et al. (1996) modeled the effects of longitudinally oriented electrodes as well as those oriented radially. They found that longitudinal orientation had little effect on threshold but a modest effect on selectivity. They also found that electrode separation had a strong effect on excitation selectivity, especially for model electrodes placed directly on the habenula.

Finally, configuration of electric stimulation (monopolar, bipolar, or tripolar) had profound effects on the threshold and spatial selectivity of evoked activity patterns. Monopolar electrodes evoked activity at the lowest current levels, but this stimulation mode evoked activity patterns that were the least selective. Bipolar electrodes evoked activity at higher current levels, but that activity was more narrowly distributed. This was especially true when the bipoles were oriented radially. Even when they were oriented longitudinally, however, bipolar electrodes could evoke selective activity patterns if they were located close together and in close contact with the osseous spiral lamina. Tripolar electrodes evoked highly 
selective activation of the auditory system. Electrodes in the tripolar configuration produced activation patterns that were nearly as restricted as patterns activated by radial VP bipolar electrodes, which in turn produced patterns that were nearly as restricted as patterns elicited by tones in normal-hearing animals. However, the currents required to evoke activity are much higher for tripolar electrodes than those required for the other two modes, except when the electrodes are very close to the modiolus. Therefore, tripolar electrode configurations may be impractical for many human CI users.

These results on the effects of stimulation mode are in good agreement with experimental results reported by previous physiological studies. Rebscher et al. (2001) reported that, in the cat, monopolar stimulation evoked ICC activity at lower thresholds with significantly broader 6-dB STC widths than radial, offradial, or longitudinal bipoles. Bierer and Middlebrooks (2002) reported that monopolar, bipolar, and tripolar stimulation evoked successively narrower STCs at successively higher thresholds in the guinea pig auditory cortex. Kral et al. (1998) compared the spatial tuning of ANFs for monopolar, longitudinal bipolar, and tripolar stimulation. They found the same sequence of selectivity and threshold as reported here, namely, a progression of increasing threshold and increasing selectivity from monopolar to tripolar stimulation. These results are in good agreement with biophysical models of ANF activation by electric stimulation. The results of Rattay (1989), Warman et al. (1992) and Briaire and Frijns (2000) all suggest that radially oriented bipolar electrodes located on the osseous spiral lamina should generate potential gradients that are steepest in a direction that is parallel with the myelinated peripheral processes of the spiral ganglion cells and least steep across the auditory nerve array.

\section{Modeling studies}

In addition to psychophysical studies of activation selectivity (Pauka 1989; Dorman et al 1990; Busby et al 1994; McDermott and McKay 1994; Nelson et al 1995; Chatterjee and Shannon 1998; McKay et al 1999; Pfingst et al 1999), several modeling studies have attempted to define the intracochlear spread of current in cochlear implants. The approaches used in these studies range from saline tank/resistive network models of voltage distributions within the cochlea (Strelioff 1973; Black and Clark 1980; Black et al. 1981, 1983; O'Leary et al. 1985; Girizon 1987; Ifukbe and White 1987; Kasper et al. 1991; Suesserman and Spelman 1993; Jolly et al. 1996; Kral et al. 1998) to 2D and 3D computer simulations of intracochlear stimulation. These computer simulations attempt to model in vivo spatial excitation patterns produced by single electrodes in the spiral of the auditory nerve array (Finley 1989; Finley et al. 1987, 1990; Frijns et al. 1994, 1995, 1996; Rattay et al. 2001a,b). Some of these models predict highly restricted distributions of intracochlear current and subsequent patterns of auditory nerve activation (e.g., Frijns et al. 1995, 1996; Finley et al. 1990; Rattay et al. 2001b), especially when the electrodes are activated as intracochlear bipolar pairs or tripolar sets. However, other models predict broad, complicated patterns of current spread in many activation modes including bipolar stimulation (Jolly et al. 1996; Ifukube and White 1987; Black et al. 1981).

\section{Significance for design of cochlear implants for humans}

Acoustic information can be encoded in the auditory nerve firing patterns using both spatial (spectral) and temporal coding. Contemporary speech processors for cochlear implants break the incoming acoustic signal into a series of frequency bands, and then extract the envelope of that signal to modulate a pulsatile carrier. This processing strategy removes most of the fine temporal information contained in the acoustic signal. Therefore, spatial coding must transmit the spectral information. The fidelity of this spatial code depends upon each intracochlear electrode selectively activating an appropriate region of the auditory nerve array. The current results suggest that some factors strongly affect the spatial selectivity of these electrodes, whereas others have little effect. For example, radial bipoles and longitudinal tripoles activate the auditory system more selectively than do longitudinal bipoles and monopoles. However, radial bipoles separated by variable distances appear to vary little in their spatial selectivity. Thus, it would appear that radial bipoles and tripoles would be the most appropriate stimulus configuration. However, these more selective configurations require higher currents to evoke a response. Since current sources are severely limited in clinical devices, activation selectivity may have to be balanced against activation efficiency. In that light, an encouraging result from the present study is that radial separation of the electrodes of a bipolar pair tended to reduce thresholds without sacrificing selectivity. It remains to be seen whether this observation can be exploited in the design of a practical implantable device.

\section{ACKNOWLEDGMENTS}

This work was supported NIH grants RO1-DC04312 and RO1-DC03549 and contracts N01-DC-7-2107, NOl-DC-2- 
1006, and P30 DC05188. We thank Chris Ellinger, Shigeto Furukawa, and Zekiye Onsan for their technical support and Brian Pfingst for his suggestions on the manuscript. Multichannel recording probes were generously provided by the University of Michigan Center for Neural Communication Technology, University of Michigan (www.engin.umich.edu/facility/cnct), which is supported by NIH/ NCRR grant P41-RR09754.

\section{REFERENCES}

Bierer JA, Middlebrooks JC. Auditory cortical images of cochlearimplant stimuli: Dependence on electrode configuration. J. Neurophysiol. 87:478-492, 2002.

Black RC, Clark GM. Differential electrical excitation of the auditory nerve. J. Acoust. Soc. Am. 67:868-874, 1980.

Black RC, Clark GM, Patrick JF. Current distribution measurements with the human cochlea. IEEE Trans. Biomed. Eng. BME-28:721-725, 1981.

Black RC, Clark GM, Tong YC, Patrick JF. Current distribution in cochlear stimulation. Ann. N.Y. Acad. Sci. 405:137-145, 1983.

BRIAIRE JJ, FrIJNS JHM. Field patterns in 3D tapered spiral model of the electrically stimulated cochlea. Hear. Res. 148:18-30, 2000.

Busby PA, Whitford LA, Blamey PJ, Richardson LM, Clark GM. Pitch perception for different modes of stimulation using the cochlear multiple-electrode prosthesis. J. Acoust. Soc. Am. 95:26582669, 1994.

Chatterjee M, Shannon RV. Forward masked excitation patterns in multielectrode electrical stimulation. J. Acoust. Soc. Am. 103:2565-2572, 1998

Collins LM, Zwolan TA, WaKefield GH. Comparison of electrode discrimination, pitch ranking, and pitch scaling data in postlingually deaf adult cochlear implant subjects. J. Acoust. Soc. Am. 101:440-455, 1997.

Dorman MF, Smith L, McCandless G, Dunnavant G, Parkin J, DanKOwskI K. Pitch scaling and speech understanding by patients who use the Ineraid cochlear implant. Ear Hear. 11:310-315, 1990.

Drake KL, Wise KD, Farraye J, Anderson DJ, BeMent SL. Performance of planar multisite microprobes in recording extracellular single-unit intracortical activity. IEEE Trans. Biomed. Eng. 35:719-732, 1988.

Eddington DK, Dobelle WH, Brackman DE, Mladejovsky MG, Parkin JL. Auditory prosthesis research with multiple channel intracochlear stimulation in Man. Ann. Otol. Rhinol. Laryngol. 87(Suppl. 53):1-39, 1978.

FINLEY CC. A finite-element model of radial bipolar field patterns in the electrically stimulated cochlea-two and three dimensional approximations and tissue sensitivities. IEEE 11th Annu. Int. Conf. Eng. Med. Biol. Soc. pp 1059-1060, 1989.

Finley CC, Wilson BS, White MW. A finite-element model of bipolar field patterns in the electrically stimulated cochlea-a two dimensional approximation. IEEE Ninth Annu. Conf. Eng. Med. Biol. Soc. 1901-1903, 1987.

Finley CC, WiLson BS, White MW. Models of neural responsiveness to electrical stimulation. In: Miller JM, Spelman FA (eds) Cochlear Implants: Models of the Electrically Stimulated Ear Springer-Verlag, New York, pp 55-96, 1990.

Frijns JHM, MooIJ J, Kate JHT. A quantitative approach to modeling mammalian myelinated nerve fibers for electrical prosthesis design. IEEE Trans. Biomed. Eng. 41:556-566, 1994.

Frijns JHM, SNoo SLD, Schoonhoven R. Potential distributions and neural excitation patterns in a rotationally symmetric model of the electrically stimulated cochlea. Hear. Res. 87:170-186, 1995.
FrIJNS JHM, SNOO SLD, KATE JHT. Spatial selectivity in a rotationally symmetric model of the electrically stimulated cochlea. Hear. Res. 95:33-48, 1996.

Furukawa S, Xu L, Middlebrooks JC. Coding of sound-source location by ensembles of cortical neurons. J. Neurosci. 20:12161228, 2000.

FuZESSERY ZM. Response selectivity for multiple dimensions of frequency sweeps in the pallid bat inferior colliculus. J. Neurophysiol. 72:1061-1079, 1994.

Girzon G. Investigation of current flow in the inner ear during electrical stimulation of intracochlear electrodes. M.S.E.E. thesis, Massachusetts Institute of Technology. Cambridge, MA, 1984.

Hanekom JJ, Shannon RV. Gap detection as a measure of electrode interaction in cochlear implants. J. Acoust. Soc. Am. 104:23722384, 1998.

Ifukube T, White RL. Current distributions produced inside and outside the cochlea from a scala tympani electrode array. IEEE Trans. Biomed. Eng. 34:883-890, 1987.

Jolly CN, Spelman FA, Clopman BM. Quadrapolar stimulation of cochlear prostheses: modeling and experimental data. IEEE Trans. Biomed. Eng. 43:857-865, 1996.

Kasper A, Pelizzoni M, Montandon P. Intracochlear potential distribution with intracochlear and extracochlear electrical stimulation in humans. Ann. Otol. Rhinol. Laryngol. 100:812-816, 1991.

Klinke R, Kral A, Heid S, Tillein J, Hartmann R. Recruitment of the auditory cortex in congenitally deaf cats by long-term cochlear electrostimulation. Science 285:1729-1733, 1999.

Kral A, Hartmann R, Mortazavi D, Klinke R. Spatial resolution of cochlear implants: The electrical field and excitation of auditory afferents. Hear. Res. 121:11-28, 1998.

Leake PA, Hradek GT, Rebscher SJ, Snyder RL. Chronic intracochlear electrical stimulation induces selective survival of spiral ganglion neurons in neonatally deafened cats. Hear. Res. 54:251-271, 1991.

Leake PA, Snyder RL, Hradek GT, Rebscher SJ. Consequences of chronic extracochlear electrical stimulation in neonatally deafened cats. Hear. Res. 82:65-80, 1995.

Leake PA, Snyder RL, Rebscher SJ, Moore CM, Vollmer M. Plasticity in central representations in the inferior colliculus induced by chronic single- vs. two-channel electrical stimulation by a cochlear implant after neonatal deafness. Hear. Res. 147:221241, 2000.

Lim HH, Tong YC, Clark GM. Forward masking patterns produced by intracochlear electrical stimulation of one and two electrode pairs in the human cochlea. J. Acoust. Soc. Am. 86:971-980, 1989.

McDermott HJ, McKay CM. Pitch ranking with non-simultaneous dual-electrode electrical stimulation of the cochlea. J. Acoust. Soc. Am. 96:155-162, 1994.

McKay CM, O'Brien A, James CJ. Effects of current level on electrode discrimination in electrical stimulation. Hear. Res. 136:159-164, 1999.

Middlebrooks JC, Bierer JA. Auditory cortical images of cochlearimplant stimuli: Coding of stimulus channel and current level. J. Neurophysiol. 87:493-507, 2002.

NaJafi K, Wise KD, Mochizuki T. A high-yield IC-compatible multichannel recording array. IEEE Trans. Electron. Dev. 32:12061211, 1985.

Nelson DA, Van Tasell DJ, Schroder AC, Soli S, Levine S. Electrode ranking of 'place pitch' and speech recognition in electrical hearing. J. Acoust. Soc. Am. 98:1987-1999, 1995.

O'Leary SJ, Black RC, Clark GM. Current distributions in the cat cochlea: A modeling and electrophysiological study. Hear. Res. 18:273-281, 1985. 
PAuKa CK. Place-pitch and vowel-pitch comparisons in cochlear implant patients using the Melbourne-Nucleus cochlear implant. J. Laryngol. Otol. Suppl. 19:1-31, 1989.

Pfingst BE, Holloway LA, Zwolan TA, Collins LM. Effects of stimulus level on electrode-place discrimination in human subjects with cochlear implants. Hear. Res. 134:105-115, 1999.

RAgGio MW, Schreiner CE. Neuronal responses in cat primary auditory cortex to electrical cochlear stimulation. III. Activation patterns in short- and long-term deafness. J. Neurophysiol. 82:3506-3526, 1999.

Rattay R. Analysis of models for extracellular fiber stimulation. IEEE Trans. Biomed. Eng. 36:676-692, 1989.

Rattay R, Richardson NL, Felix H. A model of the electrically excited cochlear neuron. I. Contribution of neural substructures to the generation and propagation of spikes. Hear. Res. 153:4363, 2001a.

Rattay R, Richardson NL, Felix H. A model of the electrically excited cochlear neuron. II. Influence of the three-dimensional cochlear structure on neural excitability. Hear. Res. 153:64-79, 2001b.

Rebscher SJ, Snyder RL, Leake PA. The effect of electrode orientation on threshold and selectivity of responses to intracochlear electrical stimulation. J. Acoust. Soc. Am. 109:2035-2048, 2001.

Schreiner CE, Langner G. Periodicity coding in the inferior colliculus of the cat. II. Topographical organization. J. Neurophysiol. 60:1823-1840, 1988.

SHANNON RV. Multi-channel electrical stimulation of the auditory nerve in man. I. Basic psychophysics. Hear. Res. 11:157-189, 1983.

ShePherd RK, BAXI JH, HARdie NA. Response of inferior colliculus neurons to electrical stimulation of the auditory nerve in neonatally deafened cats. J. Neurophysiol. 82:1363-1380, 1999.

SNYDER RL, SINEX DG. Immediate changes in tuning of cat inferior colliculus (IC) neurons following acute spiral ganglion lesions. J. Neurophysiol. 87:434-452, 2002.

Snyder RL, Rebscher SJ, Cao K, Leake PA, Kelly K. Chronic intracochlear electrical stimulation in the neonatally deafened cat. I. Expansion of central representation. Hear. Res. 50:7-33, 1990.
Snyder RL, Rebscher SJ, Leake PA, Kelly K, Cao K. Chronic intracochlear electrical stimulation in the neonatally deafened cat. II. Temporal properties of neurons in the inferior colliculus. Hear. Res. 56:246-264, 1991.

Snyder RL, Sinex DG, Walsh E, McGee J. Acute spiral ganglion lesions change the tuning and topographic organization of inferior colliculus neurons. Hear. Res. 147:200-220, 2000.

STRELIOFF D. A computer simulation of the generation and distribution of cochlear potentials. J. Acoust. Soc. Am. 54:620-629, 1973.

Suesserman M, Spelman FA. Lumped-parameter model for in vivo cochlear stimulation. IEEE Trans. Biomed. Eng. 40:237-245, 1992.

Taniguchi I, Horikawa J, Hosokawa Y, Nasu M. Optical imaging of neural activity in auditory cortex induced by intracochlear electrical stimulation. Acta Otolaryngol. Suppl. 532:83-88, 1997.

Tong YC, Clark GM. Absolute identification of electric pulse rates and electrode positions by cochlear implant patients. J. Acoust. Soc. Am. 77:1881-1888, 1985.

Tong YC, Clark GM, Blamey PJ, Busby PA, Dowell RC. Psychophysical studies for two multichannel cochlear implant patients. J. Acoust. Soc. Am. 71:153-180, 1982.

Townshend B, Cotter N, Compernolle N, White RL. Pitch perception by cochlear implant subjects. J. Acoust. Soc. Am. 82:106$115,1987$.

Van den Honert C, Stypulkowski PH. "Single fiber mapping of spatial excitation patterns in the electrically stimulates auditory nearve" Hear. Res. 29: 195-206, 1987.

Wardrop P, Rebscher SJ, Roland JT, Leake PA (2003) A temporal bone study of insertion trauma, intracochlear position of cochlear implant electrodes. I: comparison of Cochlear banded and Cochlear Contour electrodes, 2003.

Warman EN, Grill WM, Durant D. Modeling the effects of electrical fields on nerve fibers: Determination of excitation thresholds. IEEE Trans. Biomed. Eng. 39:1244-1254, 1992.

Woolsey CN, WalzL EM. Topical projections of the nerve fibers from local regions of the cochlea to the cerebral cortex of the cat. Bull. Johns Hopkins Hosp. 71:315-344, 1943. 